\title{
Cumulative readings of every do not provide evidence for events and thematic roles
}

\author{
Lucas Champollion ${ }^{\star}$ \\ University of Pennsylvania \\ Department of Linguistics, 619 Williams Hall \\ Philadelphia, PA 19104, United States \\ champoll@gmail.com
}

\begin{abstract}
An argument by Kratzer (2000) based on Schein (1986, 1993) does not conclusively show that events and thematic roles are necessary ingredients of the logical representation of natural language sentences. The argument claims that cumulative readings of every can be represented only with these ingredients. But scope-splitting accounts make it possible to represent cumulative readings of every in an eventless framework. Such accounts are motivated by obligatory reconstruction effects of every and by crosslinguistic considerations. Kratzer proposes that agent but not theme occurs in the logical representation of sentences because this allows her to model subject-object asymmetries in the distribution of cumulative every. But the reason for these asymmetries seems to be that every must be c-commanded by another quantifier in order to cumulate with it, no matter what its thematic role is. So the distribution of cumulative every does not provide support for Kratzer's proposal.
\end{abstract}

\section{Introduction}

The question whether events and thematic roles are part of the logical representation of natural language sentences has been debated for over forty years. Early formal semantic work, as well as some modern authors, simply represents the meaning of verbs with $n$ syntactic arguments as $n$-ary relations. A transitive verb, for example, is assumed to denote a two-place relation. Against this, Davidson (1967) argued that verbs denote relations between events and their arguments, so that a transitive verb denotes a three-place relation. Once events have been introduced, it becomes possible to see verbs as predicates over events, and to express the relationship between events and their arguments by separate predicates, i.e., thematic roles. This is the Neo-Davidsonian position (e.g.

\footnotetext{
* I thank Adrian Brasoveanu for sharing his insights and for stimulating discussion, which has led to many connections between our papers in this volume. My thanks also go to my advisor, Cleo Condoravdi, and to the friendly environment at PARC, particularly Danny Bobrow, Lauri Karttunen, and Annie Zaenen. I am grateful to Johan van Benthem and Eric Pacuit for the opportunity to present an early version, and to David Beaver and Eytan Zweig for discussion. After the Colloquium, Barry Schein sent detailed and thoughtful criticism, some of which is addressed in Sect. 4.
} 
Parsons, 1990; Schein, 1993). Finally, Kratzer (2000) argues for an asymmetric position, according to which only agents are represented as thematic roles. The positions are illustrated in Table 1.

Table 1. A summary of the positions in event semantics

\begin{tabular}{lll}
\hline Position & Verbal denotation & Example: Brutus stabbed Caesar \\
\hline Traditional & $\lambda y \lambda x[\operatorname{stab}(x, y)]$ & $\operatorname{stab}(b, c)$ \\
Classical Davidsonian & $\lambda y \lambda x \lambda e[\operatorname{stab}(e, x, y)] \exists e[\operatorname{stab}(e, b, c)]$ \\
Neo-Davidsonian & $\lambda e[\operatorname{stab}(e)]$ & $\exists e[\operatorname{stab}(e) \wedge \operatorname{agent}(e, b) \wedge \operatorname{theme}(e, c)]$ \\
Asymmetric & $\lambda y \lambda e[\operatorname{stab}(e, y)]$ & $\exists e[\operatorname{agent}(e, b) \wedge \operatorname{stab}(e, c)]$ \\
\hline
\end{tabular}

Over the course of the years, events and thematic roles have grown to be much more than mere notations. ${ }^{1}$ For example, many theories that resort to the thematic role agent make specific claims about the semantic content of agenthood. But the choice between the representations in Table 1 has a more basic consequence. Because they use a larger number of relations, Neo-Davidsonian and asymmetric representations offer an additional degree of freedom: They make it possible to codify meanings in which one argument's thematic role modifies a different event variable than the verb does. Such configurations are obviously impossible to write down without the help of events and thematic roles. Schein (1993) calls the property of such sentences essential separation.

The argument presented in Kratzer (2000), building on work by Schein (1986, 1993), holds that cumulative readings of every involve essential separation. While Schein's entire argument is too intricate to be addressed adequately here, my goal is to refute the specific part that relates to the word every. I will show how these readings can, in fact, be adequately captured using an eventless representation that does not use explicit roles. The crux of the argument bears on how the meaning of every is adequately represented. There are many ways to adapt eventless frameworks to the task at hand; for example, see Brasoveanu (2009) for a dynamic framework. I will stay close to the framework used in Kratzer (2000) to make the comparison as easy as possible. I will focus on the parallels with existing approaches to quantification, rather than on technical aspects.

Following Kratzer, I use the algebraic semantic framework of plurals introduced in Link (1983). ${ }^{2}$ Since Schein not only argues for events and thematic roles

\footnotetext{
${ }^{1}$ In this paper, I talk of models and logical representation languages only for convenience. I don't make any ontological claims about their existence. Readers who doubt that we should ascribe existence to models or logical representation languages in the first place should interpret the claims about whether events and thematic roles "exist" as claims about whether natural language is rich enough to express meanings which, if we choose to represent them formally, go beyond what can be expressed without using notational devices such as event variables and thematic relations.

${ }^{2}$ In algebraic frameworks, the domains of individuals and, if present, of events are each partially ordered by a mereological part-of relation $\sqsubseteq$. On the basis of $\sqsubseteq$, an operation
} 
but also, separately, against Link's framework, let me briefly justify my choice. As Schein points out, his two arguments are logically independent of each other, so his argument for events and roles can be recast in mereological terms, and this is in fact what Kratzer (2000) does. ${ }^{3}$ I have two reasons for following her example. First, this makes it easier to compare my approach to Kratzer's. Second, I will argue that cumulative readings of every can be modeled using standard accounts of cumulative readings such as Krifka (1986) and Sternefeld (1998), and these accounts happen to be formulated in Link's framework. That said, choosing this framework is not essential for my purposes as long as the domain of individuals is grounded in atoms or individuals that have no parts. Under this standard assumption, join semilattices are isomorphic to an appropriate kind of set-theoretic lattice; see Schwarzschild (1996) for an example. So everything I say about individuals can be reformulated in a set-theoretic framework.

\section{Schein's Argument as Presented in Kratzer}

Schein's case for events is very intricate and relies on complicated sentences involving three or more quantifiers. In this short paper, I only consider the part of his argument which involves cumulative readings of every. This section summarizes Kratzer's exposition of this part. It is based on the following sentence:

(1) Three copy editors caught every mistake in the manuscript.

Kratzer claims that (1) has a reading that can be paraphrased as "Three copy editors, between them, caught every mistake in the manuscript." In this reading, there are three copy editors, each of them caught at least one mistake, and every mistake was caught by at least one copy editor. ${ }^{4}$ If the subject of (1) is understood distributively, neither the surface scope reading ("Each of three copy editors caught every mistake") nor the inverse scope reading ("Each mistake is such that it was caught by each of three copy editors") is equal to Kratzer's reading, because unlike it, they both entail that each mistake was caught by more than one copy editor. One possible analysis would be to claim that in Kratzer's reading, the subject is understood collectively, so that any mistake that is caught by one of the editors counts as being caught by all three of them

$\oplus$ is defined that maps entities onto their sum, or least upper bound. $\sqsubseteq$ orders the domains of individuals and events each into a complete join semilattice; in other words, the sum operation is defined for arbitrary nonempty subsets of these domains. Singular common nouns denote predicates over atomic individuals (individuals that have no parts); plural common nouns hold of sums. The pluralization operator, written ${ }^{*}$, closes predicates $P$ under sum, i.e. ${ }^{*} P$ is the smallest set such that (i) if $P(X)$ then ${ }^{*} P(X)$; (ii) if ${ }^{*} P\left(X_{1}\right)$ and ${ }^{*} P\left(X_{2}\right)$ then ${ }^{*} P\left(X_{1} \oplus X_{2}\right)$. See Link (1998).

${ }^{3}$ For more on Schein's argument against sums, see Link (1998) and Schein (2006).

${ }^{4}$ Not all native speakers I consulted report that Kratzer's reading is in fact available from (1), though it seems present for everybody in the paraphrase that adds between them. In the following, I will grant that Kratzer's factual claim about (1) is correct. In any case, it is possible that her argument could also be based on that paraphrase, once the semantics of between them has been worked out. 
collectively (a "team credit" analysis). But, she argues, sentence (1) is true even if the editors worked independently of each other, which is incompatible with the usual understanding of the collectivity notion. In particular, (1) entails that every copy editor found at least one mistake, while collective readings do not always license this entailment. For additional arguments against a team-credit analysis, see Bayer (1997). For these reasons, I will not rely on team credit.

My strategy consists in analyzing Kratzer's reading as a cumulative reading, the kind of reading which occurs in 600 Dutch firms own 5000 American computers (Scha, 1981). It expresses that there are 600 firms and 5000 computers, each firm owns at least one computer, and each computer is owned by at least one firm. Following Krifka (1986) and Sternefeld (1998), this reading can be represented as follows, without events or thematic roles:

\section{(2) $\exists X .\left[600-\right.$ firms $(X) \wedge \exists Y$. [5000-computers $(Y) \wedge{ }^{* *}$ own $\left.\left.(X, Y)\right]\right]$.}

This representation makes use of the following ingredients and conventions. Uppercase letters are used for variables and constants that denote either atoms or sums, and lowercase letters for those that denote atoms. I use shorthands for the noun phrase denotations: for example, the predicate 600-firms is true of any sum of firms whose cardinality is 600 . The cumulation operator ${ }^{* *}$, a generalization of the pluralization operator from footnote 2 , has been defined in various ways in the literature (see e.g. Beck and Sauerland, 2000). The definition I use is from Sternefeld (1998): Given a complete join semilattice $\langle S$, $\\rangle$ and a binary relation $R \subseteq S \times S,{ }^{* *} R$ is the smallest relation such that (i) if $R(X, Y)$ then ${ }^{* *} R(X, Y)$; (ii) if ${ }^{* *} R\left(X_{1}, Y_{1}\right)$ and ${ }^{* *} R\left(X_{2}, Y_{2}\right)$ then ${ }^{* *} R\left(X_{1} \oplus X_{2}, Y_{1} \oplus Y_{2}\right)$.

Cumulative readings express information about the cardinalities of the minimal witness sets associated with the quantifiers involved (Szabolcsi, 1997). Standard representations of every have problems with this kind of configuration (Roberts, 1987). For example, interpreting "every mistake" in situ as $\lambda P . \forall x$.mistake $(x) \rightarrow P(x)$ leads to the interpretation in (3). But this is just the surface scope reading.

$$
\exists Y\left[\operatorname{three-copy-editors}(Y) \wedge \forall x\left[\operatorname{mistake}(x) \rightarrow{ }^{* *} \operatorname{catch}(Y, x)\right]\right]
$$

As Schein and Kratzer observe, if we adopt a Neo-Davidsonian position, the cumulative reading can nonetheless be represented adequately. Their idea is that once we have the agent role at our disposal, we can represent (1) roughly as "There is a sum $E$ of mistake-catching events, whose agents sum up to three editors, and every mistake was caught in at least one of these events", as in (4):

$$
\begin{aligned}
& \exists E \exists X\left[\text { three-copy-editors }(X) \wedge{ }^{* *} \operatorname{agent}(E, X)\right. \\
& \wedge \forall y[\text { mistake }(y) \rightarrow \exists e[e \sqsubseteq E \wedge \operatorname{catch}(e, y)]] \\
& \left.\wedge \exists Y\left[{ }^{*} \operatorname{mistake}(Y) \wedge{ }^{* *} \operatorname{catch}(E, Y)\right]\right]
\end{aligned}
$$

Following Schein, Kratzer takes this fact to show that we need to have at least the relation agent at our disposal in our logical representation. 


\section{Modeling Cumulative every Without Events}

The part of Schein's argument that Kratzer presents and embraces is based on the assumption that the adequate translation of every mistake is in terms of a universal quantifier. The difficulty arises from the fact that the cumulative reading of (1) expresses something about the set or sum of all mistakes. But the universal quantifier does not give us a handle on this object, because it holds of any set that contains every mistake and possibly some non-mistakes.

The first step towards a solution was taken in Landman (2000), who shifts every mistake to a referential interpretation, one that denotes the sum or group of all mistakes, written $\sigma x$.mistake $(x) .{ }^{5}$ On this view, every mistake is synonymous with the mistakes, if we disregard the fact that the latter sometimes allows nonmaximal interpretations (Krifka, 1996; Malamud, 2006).

At first sight, this suggestion faces an obvious problem: The distribution of every mistake is more restricted than the one of the mistakes. As is well known, every forces distributivity over its argument position (Kroch, 1974):

a. \#Every soldier surrounded the castle. (only distributive)

b. The soldiers surrounded the castle. (distributive or collective)

This problem can be overcome by assuming that the restrictor of every is interpreted both in its base position as a restriction on the values of its argument position, and above a distributivity $\left({ }^{*}\right)$ or cumulation $\left({ }^{* *}\right)$ operator, where it is the input to sum formation. ${ }^{6}$

Evidence that supports these assumptions comes from multiple sources. First, the assumption that it is not every itself but a separate operator that contributes distributivity is shared, in one way or another, by many authors for a variety of reasons: see e.g. Matthewson (2001), Sauerland (2003), Johnson (2007), and the papers in Szabolcsi (1997). This assumption finds crosslinguistic support in Chinese, where distributive readings require the presence of a VP modifier dou. For this reason, dou is often seen as an overt realization of the ${ }^{*}$ operator (Lee, 1986; Liu, 1990; Lin, 1998). Whenever meige, the Chinese equivalent of every, is used, the VP modifier dou must be present; and when meige originates inside the $\mathrm{VP}$, it must move out and take scope over dou, just as in the present account. ${ }^{7}$

Second, evidence that the complement of every is also interpreted in situ comes from obligatory reconstruction effects, i.e. cases in which a constituent behaves as if it was taking scope in two different places at once. Reconstruction

\footnotetext{
${ }^{5}$ Alternatively, the shift could be to a predicative interpretation, one that holds precisely of the sum of all mistakes. This solution is independently needed for variants every other and almost every which do not have a unique minimal witness. It could be exploited for explaining in terms of type mismatch why every is never interpreted in situ. For clarity of exposition, I stick to the referential interpretation of every.

6 The granularity of every is determined by its complement and not by atomicity, as pointed out by Schwarzschild (1996), using examples like Every three houses formed a block. Here, quantification is over sums of three entities, not over atomic entities. So the level of granularity is sensitive to the restrictor of every.

${ }^{7}$ Indeed, Lin (1998) makes a similar proposal for meige as Landman does for every.
} 
effects attested specifically with every are well documented in various constructions. Examples are Condition C of binding theory (Chomsky, 1993; Fox, 1999) and antecedent-contained deletion (Sauerland, 1998, 2004).

Technically, the concept that restrictors of quantifiers are interpreted in several places can be expressed in any number of ways: syntactically, for example, by creating multiple copies of phrases (Engdahl, 1986; Chomsky, 1993) or multiply dominated phrases (Johnson, 2007); or semantically, by encapsulating the contribution of the restrictor into objects that the interpretation function makes accessible in several places, such as choice functions (Sauerland, 2004) or sets of assignments (Brasoveanu, 2009). Rather than comparing all these approaches, I simply choose the proposal with the lowest types and the least departure from ordinary syntactic assumptions, both for lack of space and because this makes the interaction with the cumulation operator easier to grasp. I adopt the proposal by Fox $(1999,2002)$, according to which in situ copies are interpreted by a special semantic rule, shown here in simplified form:

(6) Trace Conversion Rule: $\llbracket[(\text { Det }) \mathrm{N}]_{i} \rrbracket=\iota y \cdot\left[\llbracket \mathrm{N} \rrbracket^{g}(y) \wedge y=g(i)\right]$

With Trace Conversion, the lower copy of a DP every $N$ which bears the index $i$ is interpreted as "the $\mathrm{N}$ which is $i$ ". The contribution of the determiner in the lower copy is ignored. Similarly to what is assumed in event semantics (Landman, 2000), I assume that all quantifiers (even those in subject position) move before they are interpreted, so that trace conversion always applies. On three copy editors, the effect of trace conversion is vacuous, so I don't show it. ${ }^{8}$

As an example, "Every dog barks" is interpreted as in (7). Here and below, the parts contributed by "every N" are underlined. The cumulative reading of (1) can be represented as in (8). ${ }^{9}$

$$
\begin{aligned}
& \underline{\sigma x \cdot \operatorname{dog}(x)} \in{ }^{*} \lambda X\left[\operatorname{barks}\left(\iota x^{\prime} \cdot \operatorname{dog}\left(x^{\prime}\right) \wedge x^{\prime}=X\right)\right] \\
& \exists X[\text { three-copy-editors }(X) \wedge \\
& \left.\langle X, \underline{\sigma y} \text {. mistake }(y)\rangle \in{ }^{* *} \lambda X^{\prime} \lambda Y\left[\operatorname{catch}\left(X^{\prime}, \underline{\iota y^{\prime}} \cdot \text { mistake }\left(y^{\prime}\right) \wedge y^{\prime}=Y\right)\right]\right]
\end{aligned}
$$

This is provably equivalent to Kratzer's representation in (4), provided that $\operatorname{catch}(x, y)$ holds whenever $\exists e[\operatorname{agent}(e, x) \wedge \operatorname{catch}(e, y)]$ and that (at least) the second argument of catch is always atomic. ${ }^{10}$ Note that the requirement that $y^{\prime}$ range over singular mistakes effectively restricts $Y$ to atomic values.

\footnotetext{
${ }^{8}$ Alternatively, one can assume in the style of Matthewson (2001) that every $N$ is interpreted as a covert variant of the partitive construction each of the $\mathrm{Ns}$, and furthermore that the $N s$ can raise out of that construction to take part in a cumulative relation. This way, subject quantifiers can be interpreted in situ.

9 The $^{* *}$ operator makes sure that the cumulated relation applies to every member of the two sums. Here, it enforces that each of the three editors was involved in catching mistakes. This avoids the "leakage" problem faced by Bayer (1997)'s account.

10 This assumption is independently necessary to model the fact that if two mistakes A and B get caught, this always implies that A gets caught and B gets caught. It is necessary for the proof because Kratzer's representation in (4) does not otherwise exclude the technical possibility that the sum event E contains some catching events in which somebody catches a sum of mistakes which do not get caught individually.
} 


\section{Mixed Cumulative-Distributive Readings}

The sentences originally discussed by Schein (1993) are more complicated than Kratzer's in several respects. For one thing, they exhibit mixed distributivecumulative configurations, such as in the following example:

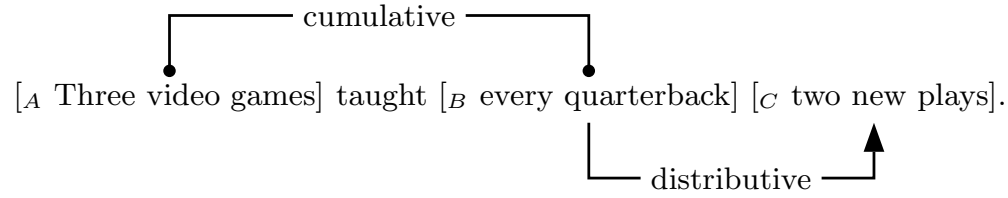

The relevant reading of this sentence is the one in which there is a given set of three videos which between them were responsible for the fact that every quarterback learned two new plays. The solution from the previous section works here as well. We can represent the reading in an eventless framework as follows:

$$
\begin{aligned}
& \exists X[\text { three-video-games }(X) \\
& \wedge\langle X, \sigma y \cdot \text { quarterback }(y)\rangle \in{ }^{* *} \lambda X^{\prime} \lambda Y[\exists Z \text { two-new-plays }(Z) \\
& \left.\left.\wedge^{* * *} \text { taught }\left(X^{\prime}, \underline{\iota y^{\prime} . q u a r t e r b a c k}\left(y^{\prime}\right) \wedge y^{\prime}=Y, Z\right)\right]\right]
\end{aligned}
$$

In this formula, the exhaustive component of "every quarterback" stands in a cumulative relation with "three video games", while its distributive component makes sure that teach relates individual quarterbacks to sums of two plays each. *** is the ternary equivalent of **. Two instances of cumulation are needed: the higher one to let three video games and every quarterback cumulate, and the lower one to reflect the lack of scopal dependency between any given set of two plays and the three video games. In other words, sentence (9) does not express for any set of two plays how many of the three video games taught that set. ${ }^{11}$

\section{Structural Asymmetries in Cumulative Readings}

Recall that Kratzer's larger goal is to argue for a representation in which only the agent role, but not the theme role, is expressed as a separate relation. Kratzer is aware that the relevant reading of (1) can be described as a cumulative reading, but she prefers not to model it as such, observing that cumulative readings are less readily available with every in general. She illustrates this with the following:

a. Three copy editors caught every mistake. $(=(1))$ Cumulative: $O K$ b. Every copy editor caught 500 mistakes. Cumulative: * c. 500 mistakes were caught by every copy editor. Cumulative: *

${ }^{11}$ Schein (1993) also considers modified numerals, which are beyond the scope of this paper. Following Schein, replacing "two" by "exactly two" in (9) entails that each quarterback was taught exactly two new plays. This is admittedly hard to represent in terms of a ternary predicate taught, because for any given quarterback, the first argument of this predicate would have to be the sum of all video games that taught him plays. Even approaches such as Krifka (1999) or Brasoveanu (2009), which deal with cumulative readings of modified numerals, do not give us access to that sum. 
Cumulative readings are absent from both (11b) and (11c). In these examples, every is in agent position. Based on this, she generalizes that every can take part in cumulative readings only when it is not in agent position, cf. (11a). This is indeed predicted by the asymmetry in her representation.

The following minimal pair is a counterexample. For Bayer (1997), (12a) is "clearly bizarre", since scripts cannot be written more than once. But he reports that $(12 \mathrm{~b})$ has a reading where every screenwriter in Hollywood contributed to the writing of the movie. We can model this by assuming that Gone with the Wind denotes a sum entity. Then if we represent (12b) as a cumulative reading as in Sect. 3, it entails that every screenwriter wrote some part of this sum.

\section{a. Every screenwriter in Hollywood wrote Gone with the Wind. \\ b. Gone with the Wind was written by every screenwriter in Holly- wood.}

Since every is in agent position in both cases, the asymmetry is unexpected on Kratzer's hypothesis. They should both be equally bizarre. This minimal pair suggests that what blocks the cumulative reading of certain every-phrases is not their thematic role. I propose instead that a noun phrase headed by every cannot cumulate with anything it c-commands. This constraint explains why every cannot cumulate in (11b) and (12a). It also predicts, contra Kratzer, that every as a passivized object noun phrase should be unable to cumulate even when it is not the agent. The following minimal pair confirms this prediction:

a. The Fijians and the Peruvians won every game. (Zweig, 2008)

b. Every game was won by the Fijians and the Peruvians.

According to Zweig, (13a) has a cumulative reading - either team won games and every game was won by one of the teams - but (13b) only has an odd distributive reading: each game was won by both teams at once. Kratzer cannot account for this contrast, since every has the same thematic role in both cases.

\section{Conclusion}

Cumulative readings of every do not pose a special problem for eventless representations, and they provide interesting evidence against its accepted translation as a generalized quantifier. The readings are not an argument that logical representations must contain events or thematic roles. The restriction on cumulative readings of every is more accurately stated in terms of c-command than in terms of thematic roles, so it is not an argument for the asymmetric account in Kratzer (2000). Of course, this does not exclude the possibility that events and thematic roles might be present in the linguistic system for other reasons. The claim here is simply that cumulative readings of every do not bear on their status.

Further work is needed to explore the c-command constraint. Interestingly, the dynamic system in Brasoveanu (2009) derives this constraint, but only with the additional assumption that cumulative every cannot take inverse scope in the sentences in question. It remains to be seen whether this assumption can be given explanatory value, given that inverse scope of every is possible in general. 


\section{Bibliography}

Bayer, S. L. (1997). Confessions of a Lapsed Neo-Davidsonian: Events and Arguments in Compositional Semantics. Garland, New York.

Beck, S. and Sauerland, U. (2000). Cumulation is needed: A reply to Winter 2000. Natural Language Semantics, 8(4):349-371.

Brasoveanu, A. (2009). Modified numerals as post-suppositions. This volume.

Chomsky, N. (1993). A minimalist program for linguistic theory. In Hale, K. and Keyser, J., editors, The View from Building 20, Essays in Linguistics in Honor of Sylvain Bromberger, pages 1-52. MIT Press.

Davidson, D. (1967). The logical form of action sentences. In Rescher, N., editor, The logic of decision and action, pages 81-95. University of Pittsburgh Press, Pittsburgh.

Engdahl, E. (1986). Constituent Questions. D. Reidel Publishing Company, Dordrecht, The Netherlands.

Fox, D. (1999). Reconstruction, binding theory, and the interpretation of chains. Linguistic Inquiry, 30(2):157-196.

Fox, D. (2002). Antecedent-contained deletion and the copy theory of movement. Linguistic Inquiry, 33(1):63-96.

Groenendijk, J., Janssen, T., and Stokhof, M., editors (1984). Truth, interpretation, information. Dordrecht: Foris.

Johnson, K. (2007). Determiners. Talk presented at On Linguistic Interfaces, Ulster.

Kratzer, A. (2000). The event argument and the semantics of verbs, chapter 2. Manuscript. Amherst: University of Massachusetts.

Krifka, M. (1986). Nominalreferenz und Zeitkonstitution. Zur Semantik von Massentermen, Pluraltermen und Aspektklassen. Fink, München (published 1989).

Krifka, M. (1996). Pragmatic strengthening in plural predications and donkey sentences. In Galloway, T. and Spence, J., editors, Proceedings of SALT 6, Ithaca. CLC Publications, Cornell University.

Krifka, M. (1999). At least some determiners aren't determiners. In Turner, K., editor, The Semantics/Pragmatics Interface from Different Points of View, pages 257-291. Elsevier.

Kroch, A. S. (1974). The semantics of scope in English. PhD thesis, Massachusetts Institute of Technology, Cambridge, Mass.

Landman, F. (2000). Events and plurality: The Jerusalem lectures. Kluwer Academic Publishers.

Lee, T. (1986). Studies on quantification in Chinese. PhD thesis, University of California at Los Angeles.

Lin, J.-W. (1998). Distributivity in Chinese and its implications. Natural Language Semantics, 6:201-243. 
Link, G. (1983). The logical analysis of plurals and mass terms: A latticetheoretical approach. In Bäuerle, R., Schwarze, C., and von Stechow, A., editors, Meaning, use and interpretation of language, pages 303-323. de Gruyter, Berlin, New York.

Link, G. (1998). Algebraic semantics in language and philosophy. Stanford: CSLI.

Liu, F.-H. (1990). Scope Dependency in English and Chinese. PhD thesis, University of California at Los Angeles.

Malamud, S. (2006). (Non-)Maximality and distributivity: a decision theory approach. In Proceedings of the 16th Conference on Semantics and Linguistic Theory (SALT 16), Tokyo, Japan.

Matthewson, L. (2001). Quantification and the nature of crosslinguistic variation. Natural Language Semantics, 9:145-189.

Parsons, T. (1990). Events in the semantics of English. MIT Press.

Roberts, C. (1987). Modal subordination, anaphora, and distributivity. $\mathrm{PhD}$ thesis, University of Massachusetts, Amherst.

Sauerland, U. (1998). The meaning of chains. PhD thesis, Massachusetts Institute of Technology, Cambridge, Massachusetts.

Sauerland, U. (2003). A new semantics for number. In The Proceedings of SALT 13, pages 258-275, Ithaca, N.Y. Cornell University, CLC-Publications.

Sauerland, U. (2004). The interpretation of traces. Natural Language Semantics, 12:63-127.

Scha, R. (1981). Distributive, collective and cumulative quantification. In Groenendijk, J., Janssen, T., and Stokhof, M., editors, Formal methods in the study of language. Mathematical Center Tracts, Amsterdam. Reprinted in Groenendijk et al. (1984).

Schein, B. (1986). Event logic and the interpretation of plurals. $\mathrm{PhD}$ thesis, Massachusetts Institute of Technology, Cambridge, MA.

Schein, B. (1993). Plurals and events. MIT Press.

Schein, B. (2006). Plurals. In Lepore, E. and Smith, B. C., editors, Oxford Handbook of Philosophy of Language. Oxford University Press, Oxford, UK.

Schwarzschild, R. (1996). Pluralities. Kluwer, Dordrecht.

Sternefeld, W. (1998). Reciprocity and cumulative predication. Natural Language Semantics, 6:303-337.

Szabolcsi, A., editor (1997). Ways of scope taking. Kluwer, Dordrecht, The Netherlands.

Zweig, E. (2008). Dependent plurals and plural meaning. PhD thesis, NYU, New York, N.Y. 